\title{
BMJ Open How do general practices respond to a pandemic? Protocol for a prospective qualitative study of six Australian practices
}

\author{
Grant Russell (D) ,1,2 Jenny Advocat, ${ }^{1}$ Riki Lane, ${ }^{1}$ Jennifer Neil, ${ }^{1}$ \\ Timothy Staunton-Smith, ${ }^{1}$ Karyn E Alexander, ${ }^{1,3}$ Simon Hattle, ${ }^{1}$ \\ Benjamin F Crabtree, ${ }^{4}$ William L Miller, ${ }^{5}$ Sumudu Neilya Setunge (D) , 1 \\ Elizabeth Ann Sturgiss (D) ${ }^{1,6}$
}

To cite: Russell G, Advocat J, Lane R, et al. How do general practices respond to a pandemic? Protocol for a prospective qualitative study of six Australian practices. BMJ Open 2021;11:e046086. doi:10.1136/ bmjopen-2020-046086

- Prepublication history and additional supplemental material for this paper are available online. To view these files, please visit the journal online (http://dx.doi.org/10.1136/ bmjopen-2020-046086).

Received 21 October 2020 Accepted 12 July 2021
Check for updates

(C) Author(s) (or their employer(s)) 2021. Re-use permitted under CC BY-NC. No commercial re-use. See rights and permissions. Published by BMJ.

For numbered affiliations see end of article.

Correspondence to Professor Grant Russell; grant.russell@monash.edu

\section{ABSTRACT}

Introduction The COVID-19 pandemic has transformed healthcare systems worldwide. Primary care providers have been at the forefront of the pandemic response and have needed to rapidly adjust processes and routines around service delivery. The pandemic provides a unique opportunity to understand how general practices prepare for and respond to public health emergencies. We will follow a range of general practices to characterise the changes to, and factors influencing, modifications to clinical and organisational routines within Australian general practices amidst the COVID-19 pandemic. Methods and analysis This is a prospective case study of multiple general practices using a participatory approach for design, data collection and analysis. The study is informed by the sociological concept of routines and will be set in six general practices in Melbourne, Australia during the 2020-2021 COVID-19 pandemic General practitioners associated with the Monash University Department of General Practice will act as investigators who will shape the project and contribute to the data collection and analysis. The data will include investigator diaries, an observation template and interviews with practice staff and investigators. Data will first be analysed by two external researchers using a constant comparative approach and then later refined at regular investigator meetings. Cross-case analysis will explain the implementation, uptake and sustainability of routine changes that followed the commencement of the pandemic.

Ethics and dissemination Ethics approval was granted by Monash University (23950) Human Research Ethics Committees. Practice reports will be made available to all participating practices both during the data analysis process and at the end of the study. Further dissemination will occur via publications and presentations to practice staff and medical practitioners.

\section{INTRODUCTION}

The COVID-19 pandemic has transformed healthcare systems worldwide as COVID-19 continues to cause high morbidity and
Strengths and limitations of this study

- This study aims to explore the ways in which general practices modify their clinical and organisational routines in response to the COVID-19 pandemic.

- We designed a qualitative, prospective case study using a participatory approach to allow a detailed, intensive exploration of individuals and organisations in context.

- A multi-methods data collection strategy comprising interviews, investigator diaries and document analysis will ensure the credibility of the findings.

- Practices are all from Melbourne Australia, an area of significant exposure to the pandemic.

mortality. ${ }^{12}$ The virus has had unprecedented health, social and economic impacts - Australia has implemented substantial economic and public health interventions to mitigate the impact of the virus. ${ }^{3}$ Primary care providers are at the forefront of the pandemic response and have needed to rapidly adjust processes and routines around service delivery. The pandemic provides a unique opportunity to understand more about how general practices prepare for and respond to public health emergencies.

At the time of writing this protocol, the majority of Australia had escaped the major public health consequences of the pandemic. The nation's relative isolation, early border closures and comprehensive physical distancing regulations had contributed to a situation where less than 100 deaths had been reported in the country during the first wave of the virus occurring between March and April 2020. ${ }^{4}$ A range of public health measures have been designed to augment traditional primary care-retired physicians and nurses have been recruited to a new 
network of government run testing centres ${ }^{5}$ and the federal government has funded 100 new general practice respiratory clinics. ${ }^{6}$

National standards ask that accredited general practices have an emergency response plan for unanticipated events such as pandemics. ${ }^{7}$ Early media responses suggest that general practitioners (GPs) have played a vital role in the pandemic response but there have been difficulties with telehealth, billing practices, the availability of personal protective equipment and a reduction in patients presenting for healthcare. ${ }^{8}$

Prior to the COVID-19 pandemic, there were very few studies that described the response of primary care in a pandemic setting. Those conducted in Australia had been overwhelmingly retrospective. ${ }^{9-11}$

This project aims to explore the ways in which general practices modify their clinical and organisational routines in response to the COVID-19 pandemic. Our qualitative case study research will draw from a range of general practices to better understand the experiences of clinicians and practice staff providing care during the pandemic. We aim to explore the practices and procedures supporting the delivery of care through answering the following research questions:

1. What changes to clinical and organisational routines have been made in general practice due to the pandemic?

2. What contextual, organisational and individual factors facilitate these changes?

\section{METHODS AND ANALYSIS}

\section{Case study and participatory approach}

We will conduct a multiple case study of general practices using a participatory approach for the design, data collection and analysis. The case study methodology uses a rapid ethnographic approach informed by the sociological concept of routines where the practice is the case. ${ }^{12}$ Routines represent patterns of interaction enacted by individuals but determined and maintained at the organisational level. ${ }^{13}$ The multiple case study approach allows a detailed, intensive exploration of individuals and organisations in context. ${ }^{14}$ Collecting varied types of data (interviews, investigator diaries, practice profiles and practice documents/signage) enhances credibility of the findings. Design was further informed by principles of participatory action research in which processes of planning, action and reflection are conducted in close collaboration with stakeholders and participants (here referring to clinical investigators and practice owners and other staff). ${ }^{15}$ To date, participatory action research has been primarily focused on empowering marginalised communities to shift the control and focus of research toward issues and concerns relevant to local needs. ${ }^{16}$ Participatory health research often involves patients, not just as subjects, but as participants in the research process. ${ }^{17} 18$

In our study, participation will involve GPs as participating investigators who will shape the project, assist with and contribute to data collection and be a part of the analysis. $^{19}$

Finally, the work will be informed by the principles of implementation science, incorporating contemporary approaches to quality improvement in primary care and being sensitive to the effects of local context on intervention delivery, to understand the process of embedding change in a practice. ${ }^{20}$

This protocol represents work to be performed after the formation of our investigator team.

\section{Context/setting}

The study will be set in six general practices in North West and South East Melbourne, Victoria, Australia and conducted between April and December 2020. Practices are all locations where GP investigators base their clinical work.

\section{Participant selection and recruitment}

Our recruitment strategy has three stages. We began by forming an investigator team comprising clinician investigators linked with the Department of General Practice at Monash University; two PhD academics with backgrounds in sociology and medical anthropology will act as external researchers (JA and RL); and two US-based primary care academics, both experienced in qualitative methods and practice based primary care research will provide advice on design, implementation and analysis as the study evolves (BFC and WLM).

In terms of recruitment GR and EAS invited potential participant investigators via email and then telephone to join the study. Each needed to be willing to participate in recruitment of the practice owner or manager and other clinicians and staff within their own practices and be responsible for components of data collection. We prioritised participants working within practices of varying size and organisational model, and aimed to recruit clinician investigators associated with between five and seven practices. We aimed to recruit between five and seven clinician investigators covering a diverse range of organisational practice models.

The second stage will involve the recruitment of general practices. Each clinician investigator will contact the practice lead/owner (or manager in the case of a community health centre) of the practices where they work. This communication will occur either through email or face to face contact. This approach will outline the concept of the study and seek practice consent to participate in the data collection.

Finally, we will recruit up to four staff members from within each practice to participate in a series of semistructured interviews over the course of the study (9 months). Members of staff will include a person in a management position in the practice (such as lead GP and/or practice manager), clinicians (GPs and practice nurses) and reception staff. In each case potential participants will first be approached by the clinician investigator 
to seek an expression of interest and consent for contact by the external researcher.

\section{Data collection}

Data collection will focus on clinical and organisational routines associated with accommodating the changes that have arisen from the 2020 COVID-19 pandemic. Consistent with the participatory approach, data will include investigator diaries, an observation template (completed by participant investigators), and interviews with practice members. Data will be collected prospectively between April 2020 and March 2021.

Written consent obtained from the practice lead/ manager will be required for the following:

1. Agreement for the clinician investigator to complete a practice description tool, modelled on those used in prior work, ${ }^{21}{ }^{22}$ at baseline and then revised at 3-6 months.

2. Photographs or copies of COVID-19-relevant signage and practice documents relating to the pandemic.

3. Consent to approach practice staff.

There will be two main data collectors per practice; the clinician investigator and the external researcher. The clinician investigator is responsible for completing the practice description tool, the participant diary and obtaining copies of relevant practice documents and photographs of practice. The external researcher is responsible for conducting in-depth interviews with members of the practice.

\section{Data collection instruments}

Our multi-method data collection strategy will use:

\section{Practice description tool}

This tool will help collate key observational and demographic data for each site. The tool will be based on a previous practice environment template. ${ }^{23}$ It will be initially completed by the clinician investigators with initial entries at baseline and then updated with ongoing collated information as the study proceeds (see online supplemental material 1.1).

\section{Interviews with clinicians, non-clinical staff and participant investigators}

An external researcher will conduct semi-structured interviews with recruited practice staff. Interviews will be conducted at a minimum of two-time points: the beginning of the study (by June 2020) and then again by November 2020. Additional interviews will be planned as required. This will be dependent on how the pandemic unfolds in the local region. This flexibility allows the study to respond to the context and enables the collection of data at potentially different stages of the pandemic.

Each clinician investigator will be interviewed in the final months of data collection, in which questions will explore key emerging findings from the case and will allow for areas of uncertainty to be clarified.

Interviews will focus on the participant's individual experience with the pandemic, perceived responses from the practices, and their thoughts on factors influencing the practice's performance with dealing with the pandemic. Interviews will last between 30 and $45 \mathrm{~min}$, follow a semi-structured template and be conducted by telephone or video-conference. These will provide additional information about the context of the interview and will assist in collecting comparable data across the regions (see online supplemental material 1.2).

\section{Investigator diaries}

Investigators will collect notes of their experiences working in general practice during the pandemic in reflective diaries collected during the study. Data collection will be focused on generating contemporaneous records of the experience from the perspective of the clinician investigator. The diary will be structured around a basic template and can be either written or collected by audio recording (in which case it will be transcribed) and will generally require entries every 1-2 weeks. The project manager will remind investigators to continue to pay attention to the diaries over the course of the study, and will collect versions of the diaries at $6-8$ weekly intervals throughout the study (see online supplemental material 1.3).

\section{Document analysis}

We will also collate practice policies and information sheets outlining practice management of the COVID-19. Documents will include the practice's prior emergency response plan and any government required plans for documenting approaches to mitigating the introduction and spread of COVID-19 in the work premises and any templates or scripts used for communication with patients and members of the community.

\section{Photographs}

Further, we will collect digital photographs of relevant practice signage, leaflets, the layout of practice waiting rooms, reception areas and any other practice structural changes that are made during the pandemic.

\section{Presentation of findings to practices}

Practices will receive a mid-project overview of findings across the practices. In the final stages of data analysis, we will share emergent findings with practices through electronic presentations of summarised practice findings. We will use a member checking procedure ${ }^{24}$ to check areas of uncertainty in the interpretation of the data. Responses to the presentation will be collected and will inform the final analysis.

\section{Data management}

Clinical and non-clinical staff interviews will be professionally transcribed and all identifying information will be removed. Interview transcripts and observational data (diaries, practice documents and field notes) will be coded using NVivo V.12. ${ }^{25}$ All digital data will be stored on a secure server only accessible by the external researchers, to protect the confidentiality of the investigators and their 
respective practices. The social scientists will be responsible for the initial coding of the data. They will work with a subgroup of the study team, comprising three of the clinician investigators, to conduct the analysis.

\section{Data analysis}

Data analysis will be conducted iteratively using a constant comparative approach. The approach will be further informed prior Canadian and Australian investigations of how primary care practice routines evolve in response to contextual change. ${ }^{12}{ }^{26}$ Data will first be analysed by RL and JA, assisted by SNS. After this, the analysis will be refined at regular investigator meetings and at a data retreat with all investigators. ${ }^{27}$

The analysis of the interview data will commence with the refinement of the existing coding template. This will be based on an initial reading and familiarisation with the raw data, as well as a priori broad theoretical concepts from Stange and Glasgow's context tool and Miller etal's relationship centred approach to primary care practice development. ${ }^{28}$ The template in the first instance will include: domains of the context tool; question clusters within each interview guide andfree text nodes.

Subsequent interview transcripts will be coded against this coding template. First cycle coding will commence against the initial coding tree. The tree will evolve following the iterative phases of qualitative data analysis.

Given the case study approach, we will use matrices to help organise and analyse the data and generate case descriptions. With our knowledge of the data and the theories underpinning the analysis we will generate a series of draft matrices, using the concepts generated by Miles, Huberman and Saldaña. ${ }^{29}$ This will be facilitated by the matrix and framework analysis functions within the NVivo software. Early matrices will be oriented to the preliminary coding template (rows), with columns being each practice. The matrices will be further informed by a series of node reports that help refine and articulate themes and concepts emerging from the data. Final, consolidated matrices will be then be used to generate COVID-19 experience narratives of 2-3 pages. These will describe the key elements of changes in each practice and facilitate the process of connection.

We will consider second cycle coding to address additional and emerging questions. In this case we will be informed by Miles' concept of second-order coding ${ }^{29}-\mathrm{a}$ process to organise and refine material from first-order coding into a more parsimonious model, with a particular focus on the emerging categorisation of causes, key relationships among participants (such as practice staff) and/ or theoretical constructs. In keeping with the iterative nature of data analysis, the decisions and details relating to this will emerge during the process of data analysis.

Finally, intervention narratives and the generated matrices will be further analysed through a process of cross-case analysis at and after the data retreat to develop hypotheses to explain the implementation, uptake and sustainability of routine changes that followed the commencement of the pandemic.

\section{Techniques to enhance trustworthiness}

We will use a variety of techniques to enhance trustworthiness in this study. Our member checking approach is described previously.

The authors have a range of experience in primary care clinical and academic practice. GR, EAS, JN, TS-S, KEA, $\mathrm{SH}$ and $\mathrm{CH}$ are all experienced primary care clinicians, each working in urban general practice in Melbourne, Australia. GR and EAS have conducted health services research in the primary care setting for 20 and 5 years, respectively. JA and RL have doctoral education in health sociology and have been working in a range of primary care-oriented studies for 10 (JA) and 7 (RL) years. BFC and WLM have worked closely together for over three decades on a series of studies investigating primary care reform in the USA and beyond. Several of these have had an international perspective and have been conducted in collaboration with GR and JA.

The Standards for Reporting Qualitative Research will be used to report the research to improve its transparency, usability and reliability. ${ }^{30}$

\section{Limitations}

The use of purposive sampling of practice staff may limit the collected data to only those individuals who are willing to disclose their perspectives on practice operations and management. Other members of staff may be reluctant to participate in the study given the investigators are members of their own practice. However, efforts will be made to protect the confidentiality of the practices as only the external researchers will have access to the initial identifiable data on a secured drive.

Patient perspectives were not sought to inform study design given the difficulties of patient interaction during a global pandemic, the need to begin data collection quickly due to the prospective design and the focus of the study being on organisational routines.

Given our qualitative case study design, the number of practices will limit the generalisability of the data. Furthermore, the use of participant investigators linked with the Department of General Practice may have influenced the focus of the study. Despite this, purposive sampling of diverse locations, multiple modes of data collection and varying sizes and organisational structures of each practice should provide trustworthy data that will increase our understanding of the key characteristics and influences of routine changes in general practice in the midst of a global health crisis.

\section{Ethics/dissemination}

Ethics approval has been obtained from the Monash University Human Research Ethics Committee (Project ID: 23950). All participants will receive complete written and verbal information about the research prior to giving full, non-coercive consent in accordance with the ethical 
guidelines. Participants and the practices in which they work are free to withdraw from participation at any time, without impacting on either their employment at the health services, or any future services.

Procedures will be followed to minimise any potential harm or distress to participants, including the provision of contact details for further assistance (available at no cost to the participant) if required. Participant privacy and confidentiality will be respected by the removal of any identifying information from data, assigning pseudonyms and storing all data safely on password-protected systems or in locked cabinets at the university. Primary data will be accessible only to JA and RL. All data will be destroyed after 7 years in accordance with the agreed ethical standards.

Beyond ethics approval, this study raises a number of ethical questions that have been considered in some detail by the investigator team. Participatory research requires what Banks et al describe as 'everyday ethics- the daily practice of negotiating the ethical issues and challenges that arise through the life of', our participatory case study. ${ }^{31}$

For many community-based participatory studies, unequal distribution of power is of major concern. Since we are collecting data from practice owners, managers and other employees, we must be mindful of the impact staff critique can have for investigators and the practice as a whole. Of these ethical concerns, most relevant for this project is the blurring of boundaries between researchers and participants and the impacts this can have on confidentiality and reliability. Numerous steps have been taken to ensure confidentiality, including ongoing reflexivity and discussion between researchers and the decision to provide access to raw data only to external researchers. Extra care will also be taken with dissemination to anonymise practices and participants, at times not using verbatim quotations or, where necessary changing certain details, given the researchers' own workplaces are the sites of the study.

Dissemination will begin from early in the data analysis when practice reports will be made available to all participating practices. At the end of the study, all practices will receive more comprehensive comparative reports. We will disseminate the results of this study via presentations at relevant local, national and international conferences, peer-reviewed journals and through social media including personal Twitter accounts and those of the Department of General Practice, Monash University, and the School of Primary and Allied Health Care. Only anonymised, non-identifiable characteristics and quotations will be used in any arising publications / reports.

\section{Public involvement statement}

Given the limitations imposed by the pandemic on interaction members of the community, this research will be carried out without patient involvement.

\section{Importance of the study}

This prospective case study is a unique opportunity to document an important moment in general practice in Australia. The use of a participatory research approach offers a promising approach to examining the challenges in and changes to general practice during the COVID-19 pandemic and the factors that facilitate these changes. The approach is particularly valuable in the midst of the pandemic - the use of clinician participant investigators eases the road to gaining informed consent, and overcomes the concerns associated with the interface between community-based research and existing government restrictions associated with the pandemic. In addition, it allows rapid tailoring of the methodology to accommodate the evolving consequences of the pandemic at a clinical, community and policy level.

As literature is emerging on the impact of the COVID-19 pandemic on primary care globally, the few international papers exploring changes to general practice have predominantly focused on the increase in telehealth consultations, ${ }^{3233}$ the impact on delayed disease diagnosis in primary care, ${ }^{34}$ the health and well-being of affected health workers, ${ }^{35} 36$ policy reviews ${ }^{37}$ and recommendations. ${ }^{38}$ Only one Belgian study, to our knowledge, has used a qualitative methodology to capture the transformation to primary care and subsequent challenges posed by the COVID-19 pandemic, as experienced by GPs. ${ }^{39}$ That study however did not examine the factors that either aided or hindered the change of practice procedures and did not incorporate the perspectives of non-clinical practice staff.

Our findings should broaden our understanding of routines in general practice, practice decision making and the ways in which practices manage an unanticipated public health emergency. The study's data collection strategy will allow us to capture the organisational process of identifying necessary adjustments in general practice routines followed by the quick implementation of new procedures and processes. We anticipate important insights into primary care training, workforce planning, and practice preparedness in the midst of an extraordinary global health challenge.

\section{Author affiliations}

${ }^{1}$ Department of General Practice, Monash University Faculty of Medicine Nursing and Health Sciences, Clayton, Victoria, Australia

${ }^{2}$ Department of Family Medicine, University of Ottawa, Ottawa, Ontario, Canada

${ }^{3}$ Altona North Medical Group, Altona North, Victoria, Australia

${ }^{4}$ Department of Family Medicine and Community Health, Research Division, Rutgers

Robert Wood Johnson Medical School, Piscataway, New Jersey, USA

${ }^{5}$ Department of Family Medicine, Leigh Valley Health Network, Allentown, Pennsylvania, USA

${ }^{6}$ National Centre for Epidemiology and Population Health, Australian National University, Canberra, Australian Capital Territory, Australia

Twitter Grant Russell @grantrussell17 and Elizabeth Ann Sturgiss @LizSturgiss Acknowledgements We would like to acknowledge the contributions of Sharon Clifford who helped coordinate the study, Zheng Zhao who provided early editorial assistance and John Furler, a clinician at one of the sites.

Contributors GR and EAS conceived the original study design. All authors helped in refining the study's final methodology. GR, EAS, JN, KEA, TS-S and SH acted as participant investigators, while BFC and WLM provided expert methodological 
advice throughout the study. This protocol paper was written by GR, JA, SNS and RL based on an earlier draft written by GR and EAS with contributions from TS-S, JN SH, BFC, WLM and KEA. All authors approved the final manuscript.

Funding The authors have not declared a specific grant for this research from any funding agency in the public, commercial or not-for-profit sectors.

Competing interests None declared.

Patient consent for publication Not required.

Provenance and peer review Not commissioned; externally peer reviewed.

Supplemental material This content has been supplied by the author(s). It has not been vetted by BMJ Publishing Group Limited (BMJ) and may not have been peer-reviewed. Any opinions or recommendations discussed are solely those of the author(s) and are not endorsed by BMJ. BMJ disclaims all liability and responsibility arising from any reliance placed on the content. Where the content includes any translated material, BMJ does not warrant the accuracy and reliability of the translations (including but not limited to local regulations, clinical guidelines, terminology, drug names and drug dosages), and is not responsible for any error and/or omissions arising from translation and adaptation or otherwise.

Open access This is an open access article distributed in accordance with the Creative Commons Attribution Non Commercial (CC BY-NC 4.0) license, which permits others to distribute, remix, adapt, build upon this work non-commercially, and license their derivative works on different terms, provided the original work is properly cited, appropriate credit is given, any changes made indicated, and the use is non-commercial. See: http://creativecommons.org/licenses/by-nc/4.0/.

\section{ORCID iDs}

Grant Russell http://orcid.org/0000-0003-3773-2355

Sumudu Neilya Setunge http://orcid.org/0000-0002-4952-1515

Elizabeth Ann Sturgiss http://orcid.org/0000-0003-4428-4060

\section{REFERENCES}

1 Newsroom WHO. COVID-19 significantly impacts health services for noncommunicable diseases, 2020. Available: https://www.who.int/ news-room/detail/01-06-2020-covid-19-significantly-impacts-healthservices-for-noncommunicable-diseases

2 van Weert H. After the first wave: what effects did the COVID-19 measures have on regular care and how can general practitioners respond to this? Eur J Gen Pract 2020;26:126-8.

3 Kidd M. Australia's primary care COVID-19 response. Aust J Gen Pract 2020;49.

4 WHO. Coronavirus (COVID-19) current situation and case numbers. 2020 13/9/20. contract No.: 14/9/20, 2020

5 AHPRA. AHPRA returns over 40,000 health practitioners to the temporary pandemic response sub-register to support our critical health workforce during the emergency. Australian Health Practitioners Regulation Agency, 2020.

6 Australian Government Department of Health. Coronavirus (COVID-19) GP respiratory clinics. Australian Government Department of Health, 2020.

7 RACGP. Standards for general practices. 5th edn. East Melbourne, Vic: The Royal Australian College of General Practitioners, 2020.

8 Smith P. Doctors are right, the lack of PPE is a scandal. AusDoc, 2020.

9 Eizenberg P. The general practice experience of the swine flu epidemic in Victoria - lessons from the front line. Med J Aust 2009;191:151-3.

10 Kunin M, Engelhard D, Thomas S, et al. Challenges of the pandemic response in primary care during Pre-Vaccination period: a qualitative study. Isr J Health Policy Res 2015;4:32.

11 Herceg A, Geysen A, Guest C, et al. SARS and biothreat preparedness--a survey of ACT general practitioners. Commun Dis Intell Q Rep 2005;29:277-82.

12 Russell G, Advocat J, Geneau R, et al. Examining organizational change in primary care practices: experiences from using ethnographic methods. Fam Pract 2012;29:455-61.

13 Becker MC. Organizational routines: a review of the literature. Industrial and Corporate Change 2004;13:643-78.
14 Patton MQ. Qualitative research \& evaluation methods. 3rd ed. Thousand Oaks, Calif: Sage Publications, 2002.

15 Meyer J. Using qualitative methods in health related action research. BMJ 2000;320:178-81.

16 Hecker R. Participatory action research as a strategy for empowering Aboriginal health workers. Aust N Z J Public Health 1997;21:784-8.

17 Colliers A, Coenen S, Philips $\mathrm{H}$, et al. Optimising the quality of antibiotic prescribing in out-of-hours primary care in Belgium: a study protocol for an action research project. BMJ Open 2017;7:e017522.

18 Bailey D, Kemp L, Wright N, et al. Talk about self-harm (TASH): participatory action research with young people, GPs and practice nurses to explore how the experiences of young people who selfharm could be improved in GP surgeries. Fam Pract 2019;36:621-6.

19 van Buul LW, Sikkens JJ, van Agtmael MA, et al. Participatory action research in antimicrobial stewardship: a novel approach to improving antimicrobial prescribing in hospitals and long-term care facilities. $J$ Antimicrob Chemother 2014;69:1734-41.

20 Coles E, Anderson J, Maxwell M, et al. The influence of contextual factors on healthcare quality improvement initiatives: a realist review. Syst Rev 2020;9:94.

21 Balasubramanian BA, Chase SM, Nutting PA, et al. Using learning teams for reflective adaptation (ultra): insights from a team-based change management strategy in primary care. Ann Fam Med 2010;8:425-32

22 Lane R, Russell G, Bardoel EA, et al. When colocation is not enough: a case study of general practitioner super clinics in Australia. Aust $J$ Prim Health 2017;23:107.

23 Ohman-Strickland PA, John Orzano A, Nutting PA, et al. Measuring organizational attributes of primary care practices: development of a new instrument. Health Serv Res 2007;42:1257-73.

24 Varpio L, Ajjawi R, Monrouxe LV, et al. Shedding the cobra effect: problematising thematic emergence, triangulation, saturation and member checking. Med Educ 2017;51:40-50.

25 QSR International Pty Ltd. NVivo qualitative data analysis software. Version 12. 2018

26 Lane R, Russell G, Bardoel EA, et al. When colocation is not enough: a case study of general practitioner super clinics in Australia. Aust $J$ Prim Health 2017;23:107-13.

27 Strauss AL. Techniques and procedures for developing grounded theory. In: Corbin JM, NetLibrary I, eds. Basics of qualitative research. 2nd edn. Thousand Oaks: Sage Publications, 1998.

28 Miller WL, Crabtree BF, Nutting PA, et al. Primary care practice development: a relationship-centered approach. Ann Fam Med 2010;8 Suppl 1:S68-79.

29 Miles MB. Qualitative data analysis. In: Huberman AM, Saldan Mŕa J, eds. A methods sourcebook. 3rd edn. Thousand Oaks, Califorinia: SAGE Publications, Inc, 2014.

30 Tong A, Sainsbury P, Craig J. Consolidated criteria for reporting qualitative research (COREQ): a 32-item checklist for interviews and focus groups. Int J Qual Health Care 2007;19:349-57.

31 Banks S, Armstrong A, Carter K, et al. Everyday ethics in communitybased participatory research. Contemp Soc Sci 2013;8:263-77.

32 Sinsky C, Linzer M. Practice and policy reset Post-COVID-19: reversion, transition, or transformation? Health Aff 2020;39:1405-11.

33 Greenhalgh T, Koh GCH, Car J. Covid-19: a remote assessment in primary care. BMJ 2020;368:m1182.

34 Jones D, Neal RD, Duffy SRG, et al. Impact of the COVID-19 pandemic on the symptomatic diagnosis of cancer: the view from primary care. Lancet Oncol 2020;21:748-50.

35 García-Sierra Rosa Ma , Badia Perich E, Manresa Dominguez Josep $\mathrm{M}^{\mathrm{a}}$. [Descriptive study of the health service workers of a Primary Care Department confined by Covid-19.]. Rev Esp Salud Publica 2020;94. [Epub ahead of print: 03 Sep 2020].

36 Lu W, Wang H, Lin Y, et al. Psychological status of medical workforce during the COVID-19 pandemic: a cross-sectional study. Psychiatry Res 2020;288:112936.

37 Huston P, Campbell J, Russell G, et al. COVID-19 and primary care in six countries. BJGP Open 2020;4. doi:10.3399/bjgpopen20X101128. [Epub ahead of print: 2710 2020].

38 Krist $\mathrm{AH}$, DeVoe JE, Cheng A, et al. Redesigning primary care to address the COVID-19 pandemic in the midst of the pandemic. Ann Fam Med 2020;18:349-54

39 Verhoeven V, Tsakitzidis G, Philips H, et al. Impact of the COVID-19 pandemic on the core functions of primary care: will the cure be worse than the disease? A qualitative interview study in Flemish GPs. BMJ Open 2020;10:e039674. 